\title{
Production by Bacillus pumilus (MSH) of an antifungal compound that is active against Mucoraceae and Aspergillus species: preliminary report
}

\author{
Edward J. Bottone and Richard W. Peluso
}

Correspondence

Edward J. Bottone

edward.bottone@mssm.edu

Received 9 April 2002

Accepted 18 August 2002
Department of Medicine, Division of Infectious Diseases, The Mount Sinai Hospital and Department of Microbiology, One Gustave L. Levy Place, Mount Sinai School of Medicine, New York, NY 10029, USA

\begin{abstract}
A compound produced by Bacillus pumilus (MSH) that inhibits Mucoraceae and Aspergillus species is described. Fungicidal activity was demonstrated by lawn-spotting and by diffusion through $0.45 \mu \mathrm{m}$ Millipore membranes placed on $5 \%$ sheep-blood agar, nutrient agar, trypticase soy agar and Mueller-Hinton agar, followed by spore inoculation of the bacterium-free underlying agar surface. With either technique, zones of fungal inhibition correlated with the zone of haemolysis produced by B. pumilus (MSH). The active compound inhibited Mucor and Aspergillus spore germination and aborted elongating hyphae, presumably by inducing a cell-wall lesion. Antifungal activity was stable in agar for a minimum of 8 days, resistant to Pronase degradation, and partially inactivated by chloroform exposure and at $\mathrm{pH}$ 5.6. Its molecular mass was determined by diffusion through dialysis membrane to be 500-3000 Da. Attempts at further isolation of the compound have proven unsuccessful to date.
\end{abstract}

\section{INTRODUCTION}

In the search for anti-infectives produced by Bacillus species, especially Bacillus cereus, Bacillus subtilis and Bacillus licheniformis, several antifungal compounds, mainly peptides, have been described (Katz \& Demain, 1977; Kugler et al., 1990; Lebbadi et al., 1994; Shosi, 1978; Silo-Suh et al., 1994). While investigating the microbial flora of a dry, used, synthetic sponge by touch inoculation to $5 \%$ sheep-blood agar, the concomitant growth of Bacillus pumilus and a Mucor species occurred after $24 \mathrm{~h}$ incubation. In areas where B. pumilus colonies had serendipitously developed either adjacent to, or in the centre of, a Mucor colony, a zone of growth inhibition of the fungus was visible. This preliminary report describes some of the biological characteristics of the $B$. pumilus antifungal compound, including its activity against Mucoraceae and Aspergillus species, partial physiochemical characteristics and a suggested mode of action.

\section{METHODS}

The producer strain was identified as Bacillus pumilus (MSH) by Gram stain, cultural characteristics and by using API 20E and API CHB strips (bioMerieux-ViTek). The isolate that served as the initial indicator strain was characterized as a Mucor species by examining slide-culture preparations, demonstrating sporangia borne on sporangiophores that arose directly from a hyphal stem cell (Richardson \& Shankland, 1995). Other bacterial and mycotic strains used to assess the spectrum of the $B$. pumilus (MSH) compound, as well as selected bacterial strains tested for potential antifungal activity, were isolated from clinical specimens. $B$. pumilus (MSH) and other bacterial test organisms were grown on $5 \%$ sheep-blood agar for $24-48 \mathrm{~h}$ at $35^{\circ} \mathrm{C}$ prior to testing, while mycotic isolates were grown on Sabouraud's agar for $48-72 \mathrm{~h}$.

Assay for antifungal activity. The lawn-spotting technique was used to determine the antimicrobial spectrum of the B. pumilus (MSH) inhibitory substance (Raubitschek \& Dostrovsky, 1950). The producer B. pumilus (MSH) strain was grown for $18-24 \mathrm{~h}$ on $5 \%$ sheep-blood agar (BBL-Microbiology Systems), after which several haemolytic colonies were selected and superimposed individually onto lawns of test mycotic and bacterial isolates. Spore suspensions of Mucoraceae and Aspergillus species were prepared by washing the growth from 4872-h-old Sabouraud agar slants with sterile distilled water. Spores were dislodged by gentle rubbing of the mycelial growth with a sterile cottontipped swab. Prior to preparing a fungal lawn, the spore suspension was examined by phase-microscopy to ensure a predominance $(>99 \%)$ of spores. Lawns were prepared by swabbing the surface of sheep-blood agar and spotting with B. pumilus (MSH). Plates were incubated at $35^{\circ} \mathrm{C}$ and examined after $24 \mathrm{~h}$. Plates showing a zone of growth inhibition were observed for 2 weeks for evidence of breakthrough growth.

To determine whether the compound could diffuse through a sterile membrane filter and remain active, $0 \cdot 45 \mu \mathrm{m}$ Millipore membrane filter was placed onto $5 \%$ sheep-blood agar and layered with an agar suspension of $B$. pumilus (MSH) prepared by inoculating a loopful of 24-h-old B. pumilus growth into melted and cooled TSA. The plates were incubated upright at $35^{\circ} \mathrm{C}$ for $18-24 \mathrm{~h}$, after which the membrane containing the agar overlay was aseptically removed and the underlying cell-free agar showing a zone of beta-haemolysis was flooded with a Mucor or Aspergillus spore suspension and examined for growth inhibition after an additional $24 \mathrm{~h}$ incubation.

The stability of the compound in agar was determined by preparing six 
membrane-diffusion plates as above. After removal of the membranes, the agar surfaces were flooded with Mucor spores at $24 \mathrm{~h}$ intervals for $96 \mathrm{~h}$, and at 144 and $168 \mathrm{~h}$, incubated and examined $24 \mathrm{~h}$ later for growth inhibition. Zone diameters were measured and recorded. While awaiting inoculation intervals, the diffusion plates were kept at $4{ }^{\circ} \mathrm{C}$.

The morphological effect of the compound on Mucor and Aspergillus was determined by microscopic $(\times 400, \times 1000)$ examination of the inhibition zone on $5 \%$ sheep-blood agar or on TSA surrounding a spotinoculum of $B$. pumilus (MSH). The zone of clearing beneath membrane filters on TSA and $5 \%$ sheep-blood agar were also examined $24-48 \mathrm{~h}$ after spore inoculation of the fungal species. Punch biopsies of these zones were also obtained for electron microscopy.

Susceptibility of the compound to Pronase $\mathrm{K}\left(10 \mathrm{mg} \mathrm{ml}^{-1}\right)$ and to chloroform was determined by flooding individual haemolytic zones of clearing beneath removed membrane filters on TSA and $5 \%$ sheepblood agar with Pronase for $1 \mathrm{~h}$, and exposing a separate set of zones to chloroform-saturated gauze for $1 \mathrm{~h}$. Afterward, the gauze was removed and the agar was aerated for $1 \mathrm{~h}$ and then both agar surfaces were flooded with Mucor spores and examined after $24 \mathrm{~h}$ for growth. Controls consisted of treating $5 \%$ sheep-blood agar with Pronase and chloroform, followed by flooding with Mucor spores.

To estimate the compound's molecular mass, sterile dialysis tubing strips with a molecular mass cut-off of $6-8 \mathrm{kDa}$ and $12-14 \mathrm{kDa}$ were placed on the surface of $5 \%$ sheep-blood agar. These were inoculated with $B$. pumilus (MSH) in agar. After $48 \mathrm{~h}$ incubation, the dialysis tubing was removed and the bacterium-free agar surface was flooded with Mucor spores, incubated for $24 \mathrm{~h}$, and examined for inhibition of Mucor.

Because the compound was not detected in overnight broth filtrates, two concentration methods were used. Initially, overnight nutrient broth and trypticase soy broth filtrates were individually dialysed against $0 \cdot 15 \mathrm{M}$ ammonium acetate using dialysis tubing with a 500 Da pore size. The dialysate was then dried by lyophilization and reconstituted in fresh sterile broth and assayed for activity by performing two-fold dilutions in nutrient broth in microtitre wells and adding $0 \cdot 1 \mathrm{ml}$ of a Mucor spore suspension to each well. After overnight incubation at $35^{\circ} \mathrm{C}$, wells were examined microscopically for growth inhibition and hyphal aberration. Controls consisted of spore-inoculation of nutrient broth alone, and using a nutrient broth filtrate of $B$. subtilis treated as above.

The second attempt at concentration included extracting overnight nutrient broth or BHIB culture filtrates by vigorous mixing with either chloroform or ethyl acetate. The resulting organic phase was then dried and the residue was dissolved and tested for activity as above. As it was strongly suspected that the antifungal activity resided in the haemolysin of the B.pumilus (MSH) producing strain, Mucor and Aspergillus species were tested against a leaky, weakly haemolytic spontaneous mutant of the parent strain as described above.

\section{RESULTS}

The B. pumilus (MSH) antifungal-producing strain grew on $5 \%$ sheep-blood agar, producing circular, adherent and sticky pearlescent colonies surrounded by a zone of betahaemolysis (Fig. 1). Colonies, which measured $3 \mathrm{~mm}$ after $48 \mathrm{~h}$, were further distinguished by their rough striated texture and three concentric rings (Fig. 1). Gram-stained smears prepared from colonies showed slender, elongated Gram-positive rods with a centrally situated ellipsoidal spore that bulged the bacillary wall. The highly motile bacterium was identified as B. pumilus (MSH) by the reactions listed in Table 1. When spot-inoculated onto sheep-blood agar

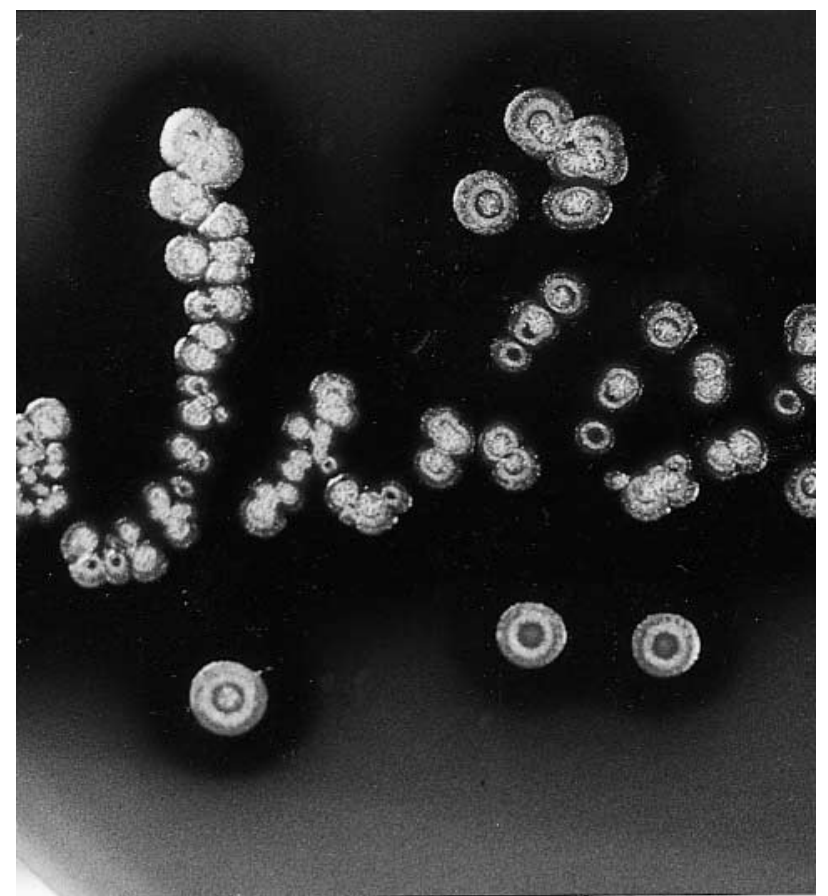

Fig. 1. Pearlescent, rough colonies of $B$. pumilus $(\mathrm{MSH})$ showing concentric rings of growth and surrounded by a zone of betahaemolysis after $48 \mathrm{~h}$ incubation on $5 \%$ sheep-blood agar.

seeded with Mucor or Aspergillus spores, a well-circumscribed clear zone of fungal growth inhibition was evident after 24 and $48 \mathrm{~h}$, respectively. Zone diameters around a $1.5 \mathrm{~cm}$ spot-inoculum of $B$. pumilus (MSH) averaged $2.2 \mathrm{~cm}$ for Mucoraceae (Mucor, Rhizopus, Cunninghamella) and $2 \mathrm{~cm}$ for Aspergillus species (Aspergillus flavus, Aspergillus fumigatus, Aspergillus niger, Aspergillus tereus, Aspergillus glacus) and coincided with the zone of beta-haemolysis produced by B. pumilus (MSH) (Fig. 2). Antifungal activity could also be demonstrated by lawn-spotting on TSA, brainheart infusion agar (BHIA), nutrient agar and MuellerHinton $(\mathrm{M}-\mathrm{H})$ agar, but not on Sabouraud's glucose agar. On the latter medium, although B. pumilus (MSH) grew readily, antifungal activity was absent, perhaps due to the low $\mathrm{pH}(5 \cdot 6)$ of the medium. The B. pumilus (MSH) inhibitory principle was fungicidal, as shown by the failure of Mucor and Aspergillus spore germination on subculture of the inhibitory zone immediately surrounding the Bacillus spot-inoculum.

The B. pumilus (MSH) inhibitory principle was inactive against Staphylococcus aureus, Streptococcus pyogenes, Streptococcus agalactiae, Enterococcus faecalis, Clostridium perfringens, B. subtilis, B. cereus, Bacillus thuringiensis, Escherichia coli, Erwinia herbicola and Klebsiella pneumoniae. Conversely, several beta haemolysin producing bacterial species including B. subtilis, B. thuringiensis, B. cereus, as well as Staphylococcus aureus, Streptococcus pyogenes and Streptococcus agalactiae failed to inhibit Mucor as tested by lawnspotting. Furthermore, an American Type Culture Collec- 
Table 1. Characteristics of the Bacillus pumilus (MSH) isolate

+, Positive; -, negative. Non-physiological characteristics: Gram-positive, slender rods and filaments, motile with ellipsoidal central spores.

\begin{tabular}{|c|c|}
\hline \multicolumn{2}{|l|}{ API 50 CHB tests } \\
\hline Glycerol & + \\
\hline Erythritol & - \\
\hline D-Arabinose & - \\
\hline L-Arabinose, ribose, D-xylose & + \\
\hline L-Xylose & - \\
\hline Adonitol & - \\
\hline$\beta$-Methyl xyloside & - \\
\hline Galactose, glucose, fructose, mannose & + \\
\hline L-Sorbose, rhamnose & - \\
\hline Dulcitol, inositol, sorbitol & - \\
\hline Mannitol & + \\
\hline $\begin{array}{l}\text { L-Methyl-D-mannoside, D-methyl-D-glucoside, } \\
N \text {-acetylglucosamine }\end{array}$ & + \\
\hline Amygdalin, arbutin, aesculin, salicin, cellobiose & + \\
\hline Maltose, lactose, sucrose, trehalose & + \\
\hline Gentiobiose, melibiose, raffinose & - \\
\hline Melezitose & - \\
\hline Starch, glycogen, inulin & - \\
\hline D-Turanose, D-tagatose & + \\
\hline D-Fucose, L-fucose, D-lyxose & - \\
\hline D-Arabitol, L-arabitol, xylitol & - \\
\hline Gluconate, 2-ketogluconate, 5-ketogluconate & - \\
\hline \multicolumn{2}{|l|}{ API $20 \mathrm{E}$ tests } \\
\hline ONPG & + \\
\hline $\mathrm{ADH}, \mathrm{LDC}, \mathrm{ODC}$ & - \\
\hline Citrate, $\mathrm{H}_{2} \mathrm{~S}$, urea, TDA, indole & - \\
\hline Voges-Proskauer & + \\
\hline Gelatin & + \\
\hline Nitrate & - \\
\hline Oxidase & + \\
\hline \multicolumn{2}{|l|}{ Additional tests } \\
\hline Catalase & + \\
\hline Growth $6.5 \% \mathrm{NaCl}$ & + \\
\hline Casein hydrolysis & + \\
\hline
\end{tabular}

tion (ATCC 7061) strain of the type species of B. pumilus, which was non-haemolytic, did not inhibit five tested isolates each of Mucoraceae and Aspergillus.

The antifungal inhibitory activity passed through $0.45 \mu \mathrm{m}$ Millipore filters placed on agar surfaces and layered with $B$. pumilus (MSH) in TSA. Removal of the filter pad after $24 \mathrm{~h}$ incubation and flooding the underlying agar surface with a Mucor or Aspergillus spore suspension resulted in a zone of fungal inhibition corresponding to the area immediately beneath the Bacillus-agar overlay. On sheep-blood agar this zone coincided with the zone of beta-haemolysis beneath the membrane (Fig. 3), whereas on blood-free media (TSA, BHIA or $\mathrm{M}-\mathrm{H}$ ) the zone of inhibition was colourless but equivalent in diameter to that on sheep-blood agar.

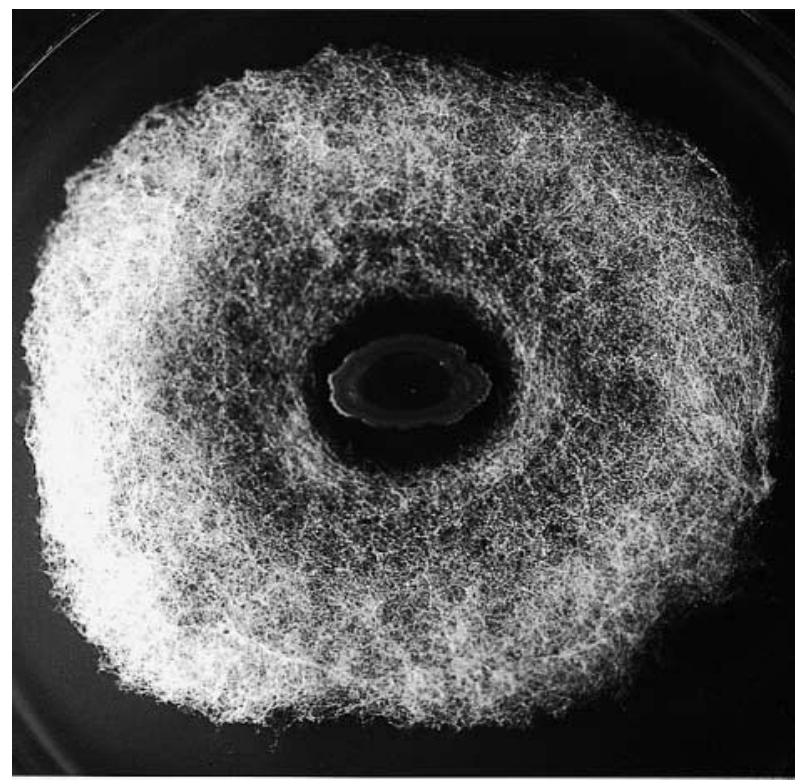

Fig. 2. Growth inhibition of Mucor species by spot-inoculation of $B$. pumilus $(\mathrm{MSH})$ onto lawn of mucor spores on $5 \%$ sheep-blood agar. Zone of inhibition coincides with zone of beta-haemolysis. Similar results were obtained for Aspergillus species.

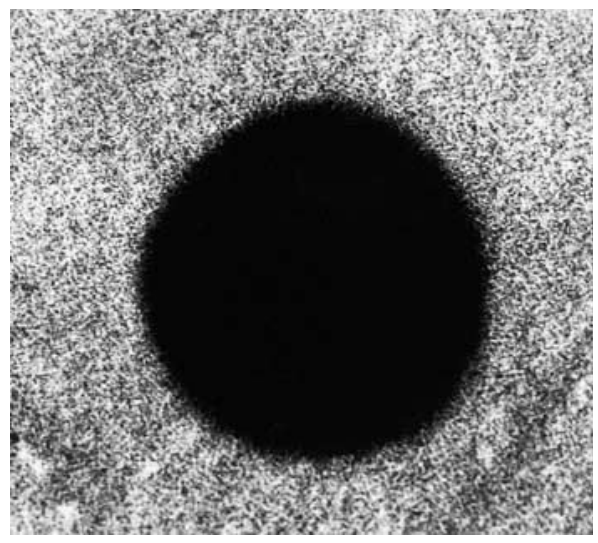

Fig. 3. Inhibition of Mucor species after diffusion of B. pumilus (MSH) antifungal through $0.45 \mu \mathrm{m}$ membrane filter on $5 \%$ sheep-blood agar. Note zone of growth inhibition coincides with zone of beta-haemolysis.

Activity was stable in agar for a minimum of 8 days as shown by only a $1 \mathrm{~mm}$ decrease in the Mucor or Aspergillus zone-ofinhibition diameter after daily flooding of individually prepared plates with Mucor or Aspergillus spores. At no time did breakthrough growth of either fungus occur.

Microscopic examination of the Mucor zone of inhibition adjacent to the B. pumilus (MSH) spot-inoculum showed a gradation in Mucor hyphal germination and elongation across the growth-inhibition zone. Immediately adjacent to 


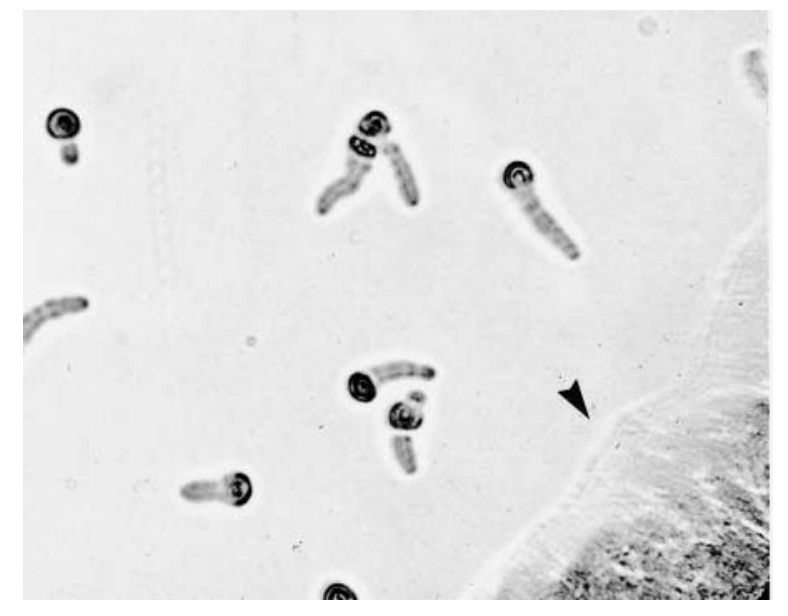

Fig. 4. Rudimentary Mucorspore germination and inhibition of hyphal elongation by $B$. pumilus (MSH) antifungal diffusing from spot inoculum (arrowhead) after $24 \mathrm{~h}$ incubation on Mueller-Hinton agar.

the spot-inoculum, rudimentary spore germination occurred with inhibition of hyphal elongation (Fig. 4). Further from the spot-inoculum, germinated hyphae were longer but were still inhibited and never traversed the inhibition zone. At the zone-of-inhibition perimeter, germinating spores oriented away from the spot-inoculum formed mycelial growth which spread away from the inhibition zone, whereas hyphae germinating from spores at the zone-of-inhibition border, but oriented so that the hyphae entered the inhibition zone, aborted as they approached the B. pumilus (MSH) spot-inoculum (Fig. 5). This same morphology was also noted on inhibition zones beneath membrane filters. Aspergillus spore germination was also rudimentary and nascent hyphae became swollen with bulbous outpocketings. Electron microscopic analysis of punch biopsies of this zone showed hyphae with apparent breaks in their chitin wall.

By growing B. pumilus (MSH) in agar on dialysis tubing, the molecular mass of the antifungal compound was estimated to be greater than $500 \mathrm{Da}$ but less than $3000 \mathrm{Da}$.

Concentrated supernatants of B. pumilus (MSH) inhibited Mucor sp. spore germination and hyphal elongation to a $1: 16$ dilution. However, as concentrated supernatants from B. subtilis, which were inactive against Mucor by lawnspotting, were also inhibitory at a $1: 8$ dilution, non-specific inhibition of Mucor was suspected. Similar results were obtained after ammonium acetate elution and concentration of BHIB and nutrient-broth filtrates.

Treatment of haemolytic zones beneath Millipore membranes with chloroform, but not Pronase, resulted in partial inactivation of the B. pumilus (MSH) antifungal activity. Germination of inoculated Mucor spores onto the chloroform-treated zone, however, required $48 \mathrm{~h}$ of incubation, whereas Mucor growth occurred within $24 \mathrm{~h}$ on chloroformtreated control plates. When the leaky B. pumilus mutant was spot-inoculated onto lawns of Mucor or Aspergillus species, zone-of-inhibition diameters were distinctly smaller $(\leqslant 1.5 \mathrm{~cm})$ as contrasted to the parent B. pumilus (MSH) $(\sim 2.5 \mathrm{~cm})$ strain.

\section{DISCUSSION}

Bacillus species produce 167 biological compounds active against bacteria, fungi, protozoa and viruses (Katz \& Demain, 1977; Cordovilla et al., 1993; Karuse et al., 1990; Berdy, 1974). Most of the antibacterials are peptides and are active against Gram-positive species while compounds such as polymyxin and colistin are active against Gram-negative

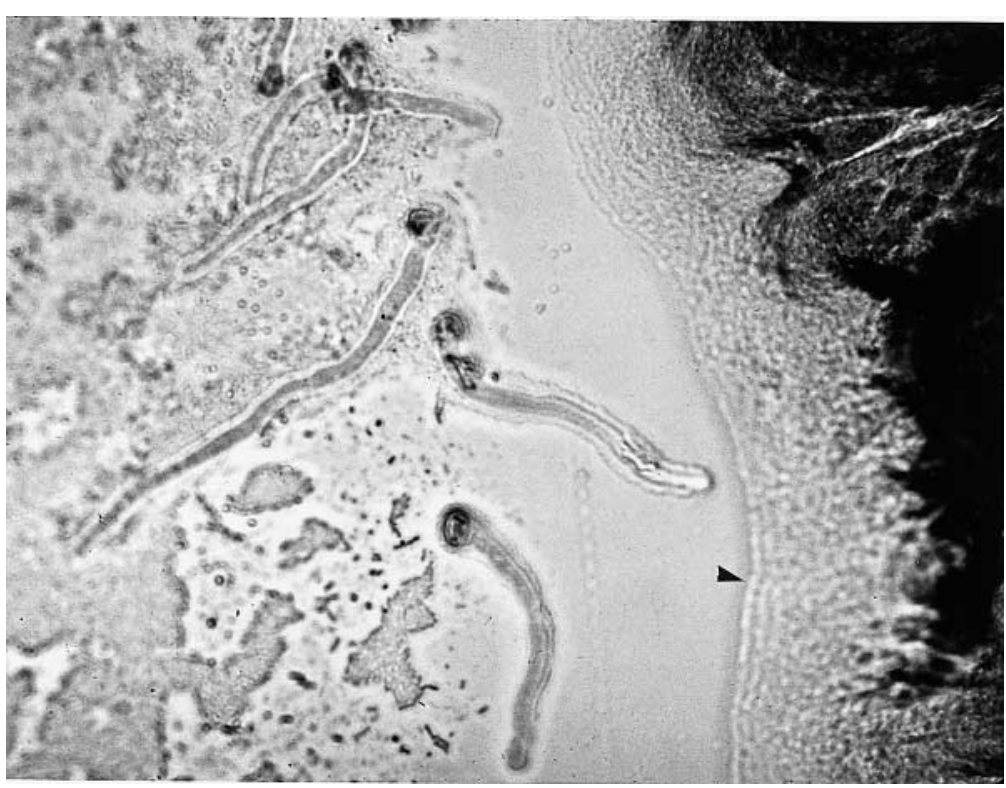

Fig. 5. Inhibition of Mucor hyphal elongation advancing toward $B$. pumilus (MSH) spot inoculum (arrowhead) and those in area of graded concentration of antifungal compound. MuellerHinton agar $24 \mathrm{~h}$ incubation. 
species. Several antifungal compounds synthesized by Bacillus species are active against filamentous fungi and yeasts. Recently, Munimbazi \& Bullerman (1998a) described antifungal metabolites produced by $B$. pumilus grown in potato glucose broth, which inhibited mycelial growth (spore germination) of Aspergillus, Penicillium and Fusarium species, and aflatoxin production by Aspergillus parasiticus (Munimbazi \& Bullerman, 1998b). There are, however, several differences between our B. pumilus (MSH) antifungal compound and that described by Munimbazi \& Bullerman (1998a). B. pumilus (MSH) produces an antifungal metabolite that inhibits the growth of Mucoraceae and Aspergillus species including A. flavus, A. fumigatus and A. terreus. While no data were given by Munimbazi \& Bullerman (1998a) regarding Mucoraceae, their B. pumilus compound was inactive against $A$. flavus and A. terreus, and was not tested against $A$. fumigatus. Additionally, in contrast to the $B$. pumilus metabolite found by Munimbazi \& Bullerman (1998a), the B. pumilus (MSH) product does not inhibit Fusarium species.

Mechanistically, the B. pumilus (MSH) metabolite inhibits both spore germination and hyphal elongation, as well as newly germinated advancing hyphae. Preliminary electron microscopy of inhibited germinating hyphae suggests a cellwall lesion that could lead to leakage of intracellular components and hyphal death. Such a mechanism of action could also account for inhibition of advancing hyphae upon contact with the $B$. pumilus (MSH) antifungal compound. The B. pumilus strain described by Munimbazi \& Bullerman (1998a) inhibits spore germination without mention of hyphal inhibition.

The B. pumilus (MSH) antifungal principle appears to equate to the haemolysin produced by this strain, which is corroborated by a reduction in the zone of antifungal activity by the isogenic haemolysin-deficient $B$. pumilus and the complete absence of antifungal activity by the type strain of B. pumilus, ATCC $7061^{\mathrm{T}}$, which is non-haemolytic.

Our metabolite was inactive when tested on Sabouraud's glucose agar, which has a $\mathrm{pH}$ of $5 \cdot 6$, in contrast to that of Munimbazi \& Bullerman (1998a) whose metabolite was active over a $\mathrm{pH}$ range of $2-10$. Pronase treatment of haemolytic zones beneath membrane filters did not inactivate activity whereas chloroform exposure resulted in partial inactivation as manifested by breakthrough germination of Mucor spores $48 \mathrm{~h}$ after chloroform treatment. Taken in concert with an estimated molecular mass of between 500 and $3000 \mathrm{Da}$, our compound resembles other peptide antifungals produced by Bacillus species (Katz \& Demain, 1977; Lebbadi et al., 1994; Wakayama et al., 1984), but it may also contain a lipid moiety.

Although our compound is stable in agar for a minimum of 8 days, we have been unable to isolate it in sufficient concentrations to perform activity, cytotoxicity and more definitive physiochemical studies. Perhaps the active molecule (haemolysin) requires a stabilizing carrier molecule, e.g. erythro- cyte, spore/hyphae or agar, to maintain activity. When dissociated, activity is lost. The ability of the compound to remain active (stable) for more than 8 days in agar supports this concept.

\section{Conclusions}

We have described a compound produced by B. pumilus (MSH) that has fungicidal activity against Mucoraceae and Aspergillus spp. The compound is of low molecular mass and inhibits spore germination and hyphal elongation, rendering it versatile in potential prophylaxis against, and treatment of, opportunistic infections caused by the above angioinvasive fungal pathogens. Purification and molecular characterization of the compound could lead to development of an innovative antifungal in an area of clinical need. The B. pumilus (MSH) strain was deposited in the American Type Culture Collection as ' $B$. sinai' ATCC 55692 prior to our obtaining a species designation, and has been accorded United States Patent Number $6,090,613$.

\section{ACKNOWLEDGEMENTS}

The authors wish to thank Wilson A. Quezada, presently a medical student at Columbia University, who as a high school student, provided dedicated technical assistance.

\section{REFERENCES}

Berdy, J. (1974). Recent developments of antibiotic research and classification of antibiotics according to chemical structure. Adv Appl Microbiol 18, 309-406.

Cordovilla, P., Valdivia, E., Gonzalez-Segura, A., Galvez, A., MartinezBueno, M. \& Maqueda, M. (1993). Antagonistic action of the bacterium Bacillus licheniformis M-4 toward the amoeba Naegleria fowleri. J Eukaryot Microbiol 40, 323-328.

Karuse, N., Tenyo, O., Kobaru, S., Kamei, H., Miyaki, T., Konishi, M. \& Oki, T. (1990). Pumilucidin, a complex of new antiviral antibiotics. Production, isolation, chemical properties, structure and biological activity. J Antibiot 43, 267-280.

Katz, E. \& Demain, A. L. (1977). The peptide antibiotics of Bacillus: chemistry, biogenesis and possible functions. Bacteriol Rev 40, 449-474.

Kugler, M., Loeffler, W., Rapp, C., Kern, A. \& Jung, A. G. (1990). Rhizocticin A, an antifungal phosphono-oligopeptide of Bacillus subtilis ATCC 6633: biological properties. Arch Microbiol 153, 276-281.

Lebbadi, M., Galvez, A., Maqueda, M., Martinez-Bueno, M. \& Valihuia, E. (1994). Fungicin M4: a narrow spectrum peptide antibiotic from Bacillus licheniformis M-4. J Appl Bacteriol 77, 49-53.

Munimbazi, C. \& Bullerman, L. B. (1998a). Isolation and partial characterization of antifungal metabolites of Bacillus pumilus. J Appl Microbiol 84, 959-968.

Munimbazi, C. \& Bullerman, L. B. (1998b). Inhibition of aflatoxin production of Aspergillus parasiticus NRRL 2999 by Bacillus pumilus. Mycopathologia 140, 163-169.

Raubitschek, F. \& Dostrovsky, A. (1950). An antibiotic against dermatophytes, derived from Bacillus subtilis. Dermatologia 100, 1-4.

Richardson, M. D. \& Shankland, G. S. (1995). Rhizopus, Rhizomucor, Absidia and other agents of systemic and subcutaneous zygomycoses. In 
Manual of Clinical Microbiology, 6th edn, pp. 809-846. Edited by P. R. Murray, E. J. Baron, M. A. Phaller, F. C. Tenover \& R. H. Yolken. Washington, DC: ASM Press.

Shosi, J. (1978). Recent chemical studies on peptide antibiotics from the genus Bacillus. Adv Appl Microbiol 24, 187-214.
Silo-Suh, L. A., Lethbridge, B. J., Raffel, S. J., Clardy, J. \& Handelsman, J. (1994). Biological activities of two fungistatic antibiotics produced by Bacillus cereus UW 85. Appl Environ Microbiol 60, 2023-2030.

Wakayama, S., Ishikawa, F. \& Oishi, K. (1984). Mycocerein, a novel antifungal peptide antibiotic produced by Bacillus cereus. Antimicrob Agents Chemother 26, 939-940. 